Supporting information for:

\title{
Diene-Titanium Complexes as Synthetic Intermediates for the Construction of Three- or Five-Membered Carbocycles
}

Philippe Bertus, ${ }^{\mathrm{a}, \mathrm{b}}$ Christine Menant, ${ }^{\mathrm{a}}$ Chloé Tanguy ${ }^{\mathrm{a}}$ and Jan Szymoniak ${ }^{\mathrm{a}}$

${ }^{a}$ UMR 6519 - RSA, CNRS - Université de Reims Champagne-Ardenne, BP

1039, 51687 Reims Cedex 2, France.

${ }^{\mathrm{b} U M R} 6011$ - UCO2M, CNRS - Université du Maine, 72085 Le Mans

cedex 9, France.

philippe.bertus@univ-lemans.fr, jan.szymoniak@univ-reims.fr

\section{$\underline{\text { I - General }}$}

All reactions were conducted under an atmosphere of dry argon using standard Schlenk techniques. Diethylether and THF were distilled from sodium benzophenone ketyl prior to use. $\mathrm{Ti}(\mathrm{O} i \text {-Pr })_{4}$ was used as received. Grignard reagents were prepared in $\mathrm{Et}_{2} \mathrm{O}$ from the corresponding bromide, and titrated in THF by menthol in the presence of orthophenanthroline. Cyanoesters 3a-c and cyanocarbonate 3d were prepared following reported procedures. ${ }^{1}$ Flash chromatography was performed with silica gel 60 (Merck, 40-63 $\mu \mathrm{m}) .{ }^{1} \mathrm{H}$ and ${ }^{13} \mathrm{C}$ NMR spectra were recorded in $\mathrm{CDCl}_{3}$ on a Bruker $\mathrm{AC}-250$ or DRX-500 spectrometer. Chemical shifts were measured in $\delta(\mathrm{ppm})$ and coupling constants $J$ in $\mathrm{Hz}$ (solvent peak reference: $\delta=7.26$ for ${ }^{1} \mathrm{H}, 77.0$ for ${ }^{13} \mathrm{C}$ ). IR spectra were recorded on a Nicolet Avatar 320 instrument. Mass spectra were recorded on a ThermoFinnigan Trace MS spectrometer. High Resolution Mass Spectra (HRMS) were performed on Q-TOF Micro micromass positive $\mathrm{ESI}(\mathrm{CV}=30 \mathrm{~V})$.

\section{2. - Preparation of amines 1a-e}

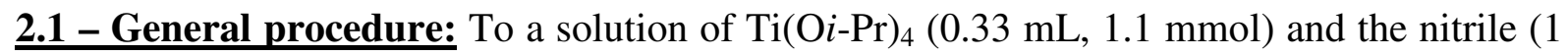
$\mathrm{mmol})$ in $\mathrm{Et}_{2} \mathrm{O}(5 \mathrm{~mL})$ was added under argon at $\mathrm{rt}$ a solution of the homoallylic Grignard reagent (2.2 mmol, 0.5-1 $\mathrm{M}$ in $\mathrm{Et}_{2} \mathrm{O}$ ). The mixture was stirred during $1 \mathrm{~h}$, then $1 \mathrm{~N} \mathrm{HCl}$ (ca 3 $\mathrm{mL}$ ) and ethyl acetate (ca $15 \mathrm{~mL})$ were added. $\mathrm{NaOH}(10 \%$ aq, ca $10 \mathrm{~mL})$ was added and the

(1) (a) Laroche, C.; Harakat, D.; Bertus, P.; Szymoniak, J. Org. Biomol. Chem. 2005, 3, 3482-3487. (b) Carpino, L. A. J. Am. Chem. Soc. 1962, 84, 2196-2201. 
mixture was extracted with ethyl acetate. The combined organic layers were dried $\left(\mathrm{MgSO}_{4}\right)$, filtered, and concentrated under reduced pressure. The residue was purified by flash chromatography on silica gel $\left(\mathrm{Et}_{2} \mathrm{O}, \mathrm{Et}_{2} \mathrm{O} / \mathrm{Et}_{3} \mathrm{~N}\right.$ 98:2).

\section{2 - (1-Phenylcyclopent-3-enyl)amine (1a)}

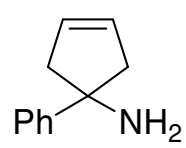

Pale yellow oil.

IR (film): $v\left(\mathrm{~cm}^{-1}\right)$ 3353, 1600, 1493, 1446.

${ }^{1} \mathrm{H}$ NMR (250 MHz, $\left.\mathrm{CDCl}_{3}\right): \delta(\mathrm{ppm}) 1.72(\mathrm{br} \mathrm{s}, 2 \mathrm{H}), 2.42(\mathrm{~d}, J=15.6 \mathrm{~Hz}, 2 \mathrm{H}), 2.79(\mathrm{~d}, J=$ 15.6 Hz, $2 \mathrm{H}), 5.76$ (s, $2 \mathrm{H})$, 7.05-7.32 (m, $5 \mathrm{H})$.

${ }^{13} \mathrm{C} \mathrm{NMR}\left(\mathrm{CDCl}_{3}, 63 \mathrm{MHz}\right): \delta(\mathrm{ppm}) 50.2\left(2 \mathrm{CH}_{2}\right), 62.3(\mathrm{C}), 125.2(2 \mathrm{CH}), 126.1(2 \mathrm{CH})$, $128.1(2 \mathrm{CH}), 128.7(2 \mathrm{CH}), 149.2(\mathrm{C})$.

MS (EI): m/z (\%) $159\left(\mathrm{M}^{+\bullet}, 49\right), 158$ (83), 104 (75), 77 (100).

HRMS (ES): m/z (\%) $160.1128\left(\mathrm{MH}^{+} \mathrm{C}_{11} \mathrm{H}_{14} \mathrm{~N}\right.$ requires 160.1128).

\section{$\underline{2.3 \text { - (3-Methyl-1-phenylcyclopent-3-enyl)amine (1b) }}$}

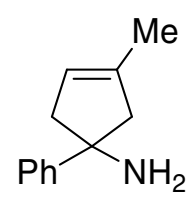

Pale yellow oil.

IR (film): $v\left(\mathrm{~cm}^{-1}\right) 3353,3283,1601,1494,1445$.

${ }^{1} \mathrm{H}$ NMR (250 MHz, $\mathrm{CDCl}_{3}$ ): $\delta$ (ppm) 1.79 (br s, $\left.5 \mathrm{H}\right), 2.40-2.56(\mathrm{~m}, 2 \mathrm{H}), 2.84-2.95(\mathrm{~m}, 2 \mathrm{H})$, 5.38 (br s, $1 \mathrm{H}), 7.21-7.48$ (m, $5 \mathrm{H})$.

${ }^{13} \mathrm{C}$ NMR $\left(\mathrm{CDCl}_{3}, 63 \mathrm{MHz}\right): \delta(\mathrm{ppm}) 16.8\left(\mathrm{CH}_{3}\right), 50.5\left(\mathrm{CH}_{2}\right), 54.3\left(\mathrm{CH}_{2}\right), 63.5(\mathrm{C}), 122.2$ (CH), 125.1 (2 CH), $126.1(\mathrm{CH}), 128.1(2 \mathrm{CH}), 138.3(\mathrm{C}), 149.7(\mathrm{C})$.

MS (EI): m/z (\%) $173\left(\mathrm{M}^{+\bullet}, 100\right), 158$ (88), 143 (43), 132 (40), 104 (80), 91 (35).

HRMS (ES): $\mathrm{m} / \mathrm{z}(\%) 174.1288\left(\mathrm{MH}^{+} \mathrm{C}_{12} \mathrm{H}_{16} \mathrm{~N}\right.$ requires 174.1283).

\section{$\underline{2.4-(1-n o n y l c y c l o p e n t-3-e n y l) a m i n e ~(1 c) ~}$}

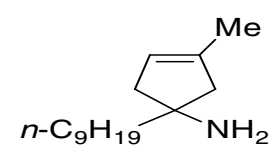

Pale yellow oil. 
IR (film): $v\left(\mathrm{~cm}^{-1}\right)$ 3354, 2925, 2853, 1466.

${ }^{1} \mathrm{H}$ NMR $\left(250 \mathrm{MHz}, \mathrm{CDCl}_{3}\right): \delta(\mathrm{ppm}) 0.81(\mathrm{t}, J=6.4 \mathrm{~Hz}, 3 \mathrm{H}), 1.10-1.50(\mathrm{~m}, 18 \mathrm{H}), 2.08(\mathrm{~d}$, $J=15.1 \mathrm{~Hz}, 2 \mathrm{H}), 2.32(\mathrm{~d}, J=15.1 \mathrm{~Hz}, 2 \mathrm{H}), 5.60$ (s, $2 \mathrm{H})$.

${ }^{13} \mathrm{C} \mathrm{NMR}\left(\mathrm{CDCl}_{3}, 63 \mathrm{MHz}\right): \delta(\mathrm{ppm}) 14.0\left(\mathrm{CH}_{3}\right), 22.6\left(\mathrm{CH}_{2}\right), 24.9\left(\mathrm{CH}_{2}\right), 29.2\left(\mathrm{CH}_{2}\right), 29.5$ $\left(\mathrm{CH}_{2}\right), 29.6\left(\mathrm{CH}_{2}\right), 30.2\left(\mathrm{CH}_{2}\right), 31.8\left(\mathrm{CH}_{2}\right), 42.8\left(\mathrm{CH}_{2}\right), 47.8\left(\mathrm{CH}_{2}\right), 60.3(\mathrm{C}), 128.8(\mathrm{CH})$.

MS (EI): m/z (\%) $209\left(\mathrm{M}^{+\bullet}, 44\right), 154$ (40), 110 (70), 82 (100).

HRMS (ES): $\mathrm{m} / \mathrm{z}(\%) 210.2213\left(\mathrm{MH}^{+} \mathrm{C}_{14} \mathrm{H}_{28} \mathrm{~N}\right.$ requires 210.2222).

\section{$\underline{2.5 \text { - (3-Methyl-1-nonylcyclopent-3-enyl)amine (1d) }}$}

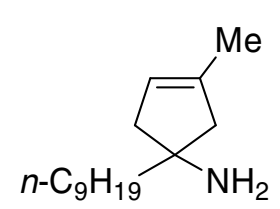

Pale yellow oil.

IR (film): $v\left(\mathrm{~cm}^{-1}\right)$ 3355, 2924, 2853, 1466, 1377.

${ }^{1} \mathrm{H}$ NMR $\left(250 \mathrm{MHz}, \mathrm{CDCl}_{3}\right): \delta(\mathrm{ppm}) 0.85(\mathrm{t}, J=6.8 \mathrm{~Hz}, 3 \mathrm{H}), 1.20-1.35(\mathrm{~m}, 18 \mathrm{H}), 1.70(\mathrm{~s}, 3$ H), 1.97-2.12 (m, 2 H), 2.30-2.46 (m, 2 H), 5.25 (br s, 1 H).

${ }^{13} \mathrm{C} \mathrm{NMR}\left(\mathrm{CDCl}_{3}, 63 \mathrm{MHz}\right): \delta(\mathrm{ppm}) 13.9\left(\mathrm{CH}_{3}\right), 16.7\left(\mathrm{CH}_{3}\right), 22.5\left(\mathrm{CH}_{2}\right), 24.7\left(\mathrm{CH}_{2}\right), 29.2$ $\left(\mathrm{CH}_{2}\right), 29.4\left(\mathrm{CH}_{2}\right), 29.5\left(\mathrm{CH}_{2}\right), 30.1\left(\mathrm{CH}_{2}\right), 31.7\left(\mathrm{CH}_{2}\right), 42.9\left(\mathrm{CH}_{2}\right), 47.9\left(\mathrm{CH}_{2}\right), 51.8\left(\mathrm{CH}_{2}\right)$, $60.8(\mathrm{C}), 122.1(\mathrm{CH}), 138.1(\mathrm{C})$.

MS (EI): m/z (\%) $223\left(\mathrm{M}^{+\bullet}, 16\right), 154$ (48), 124 (84), 96 (100).

HRMS (ES): $\mathrm{m} / \mathrm{z}(\%) 214.2381\left(\mathrm{MH}^{+} \mathrm{C}_{15} \mathrm{H}_{30} \mathrm{~N}\right.$ requires 214.2378).

\section{$\underline{2.6-(1-B e n z y l-3-m e t h y l c y c l o p e n t-3-e n y l) a m i n e ~(1 e) ~}$}

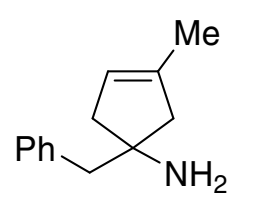

Pale yellow oil.

IR (film): $v\left(\mathrm{~cm}^{-1}\right)$ 3353, 1639, 1602, 1581, 1495, 1453.

${ }^{1} \mathrm{H}$ NMR (250 MHz, $\mathrm{CDCl}_{3}$ ): $\delta$ (ppm) 1.43 (br s, $\left.2 \mathrm{H}\right), 1.71$ (s, $\left.3 \mathrm{H}\right), 1.93-2.10$ (m, $\left.2 \mathrm{H}\right), 2.48$ 2.59 (m, 2 H), 2.84 (s, 2 H), 5.26 (br s, 1 H), 7.16-7.31 (m, 5 H).

${ }^{13} \mathrm{C}$ NMR $\left(\mathrm{CDCl}_{3}, 63 \mathrm{MHz}\right): \delta(\mathrm{ppm}) 16.9\left(\mathrm{CH}_{3}\right), 47.6\left(\mathrm{CH}_{2}\right), 48.9\left(\mathrm{CH}_{2}\right), 51.5\left(\mathrm{CH}_{2}\right), 61.4$ (C), $122.2(\mathrm{CH}), 126.2(\mathrm{CH}), 128.0(2 \mathrm{CH}), 130.1(2 \mathrm{CH}), 138.3(\mathrm{C}), 138.6(\mathrm{C})$.

MS (EI): m/z (\%) $187\left(\mathrm{M}^{+\bullet}, 9\right), 172$ (26), 129 (15), 96 (100). 
HRMS (ES): $\mathrm{m} / \mathrm{z}(\%) 188.1443\left(\mathrm{MH}^{+} \mathrm{C}_{13} \mathrm{H}_{18} \mathrm{~N}\right.$ requires 188.1439).

\section{3. - Preparation of compounds 4a-f}

3.1 - General procedure: To a solution of $\operatorname{Ti}(\mathrm{O} i-\mathrm{Pr})_{4}(0.33 \mathrm{~mL}, 1.1 \mathrm{mmol})$ and the functionalized nitrile 3a-d $(1 \mathrm{mmol})$ in the specified solvent (see Table 1, $5 \mathrm{~mL}$ ) was added

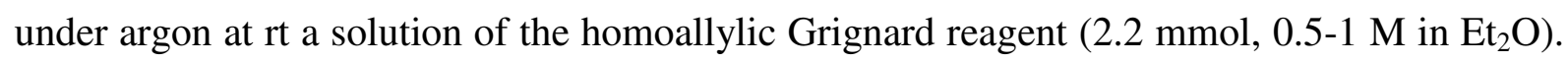
The mixture was stirred during $1 \mathrm{~h}$, then water $(\mathrm{ca} 1 \mathrm{~mL})$ and ethyl acetate $(10 \mathrm{~mL})$ were added, giving a white precipitate on the wall of the flask. The mixture was extracted twice with ethyl acetate. The combined organic layers were dried $\left(\mathrm{MgSO}_{4}\right)$, filtered, and concentrated under reduced pressure. The residue was purified by flash chromatography on silica gel (EtOAc or $\left.\mathrm{Et}_{2} \mathrm{O}\right)$.

\section{2 - 4-Aza-1-vinylspiro[2.4]heptan-5-one (4a)}

Reaction performed in $\mathrm{Et}_{2} \mathrm{O}: 65 \%$ yield (85:15 mixture of diastereomers)

Reaction performed in THF: $72 \%$ yield (98:2 mixture of diastereomers)

The two diastereomers can be separated by chromatography (silica gel $-\mathrm{CH}_{2} \mathrm{Cl}_{2} / i$ - $\mathrm{PrOH}$ 95:5)

$$
\underline{\left(1 R^{*}, 3 R^{*}\right)-4 a}
$$

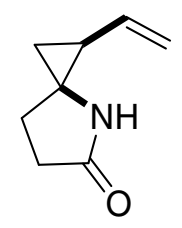

White solid. Mp $100-102{ }^{\circ} \mathrm{C}$

$\mathrm{R}_{f} 0.49$ (Silica gel, $\mathrm{CH}_{2} \mathrm{Cl}_{2} / \mathrm{i}-\mathrm{PrOH}$ 95:5)

IR (KBr): $v\left(\mathrm{~cm}^{-1}\right)$ 3184, 3074, 1696, 1658, 1632, 1370.

${ }^{1} \mathrm{H}$ NMR $\left(250 \mathrm{MHz}, \mathrm{CDCl}_{3}\right): \delta(\mathrm{ppm}) 0.87(\mathrm{t}, J=6.4 \mathrm{~Hz}, 1 \mathrm{H}), 0.95(\mathrm{dd}, \mathrm{J}=9.2,6.3 \mathrm{~Hz}, 1 \mathrm{H})$, $1.53(\mathrm{td}, \mathrm{J}=9.2,6.5 \mathrm{~Hz}, 1 \mathrm{H}), 2.01(\mathrm{ddd}, \mathrm{J}=12.8,9.4,5.9 \mathrm{~Hz}, 1 \mathrm{H}), 2.10-2.21(\mathrm{~m}, 1 \mathrm{H}), 2.30$ $2.53(\mathrm{~m}, 2 \mathrm{H}), 4.99$ (dd, J = 10.2, $1.7 \mathrm{~Hz}, 1 \mathrm{H}), 5.06$ (ddd, J = 17.0, 1.7, 0.6 Hz, $1 \mathrm{H}), 5.51$ (ddd, J = 17.0, 10.2, 8.8 Hz, $1 \mathrm{H}), 8.15$ (br s, $1 \mathrm{H}$ ).

${ }^{13} \mathrm{C}$ NMR $\left(63 \mathrm{MHz}, \mathrm{CDCl}_{3}\right): \delta(\mathrm{ppm}) 17.3\left(\mathrm{CH}_{2}\right), 27.2(\mathrm{CH}), 29.8\left(\mathrm{CH}_{2}\right), 30.9\left(\mathrm{CH}_{2}\right), 44.9$ (C), $115.0\left(\mathrm{CH}_{2}\right), 135.8(\mathrm{CH}), 178.6(\mathrm{C})$.

MS (EI): m/z (\%) 137 (M+•, 100), 108 (22), 94 (50), 80 (24), 53 (73).

HRMS (ES): m/z (\%) $160.0737\left(\mathrm{MNa}^{+} \mathrm{C}_{8} \mathrm{H}_{11} \mathrm{NONa}\right.$ requires 160.0738). 


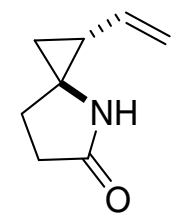

Colorless oil.

$\mathrm{R}_{f} 0.43$ (Silica gel, $\mathrm{CH}_{2} \mathrm{Cl}_{2} / i-\mathrm{PrOH}$ 95:5)

${ }^{1} \mathrm{H}$ NMR $\left(250 \mathrm{MHz}, \mathrm{CDCl}_{3}\right): \delta(\mathrm{ppm}) 0.78(\mathrm{t}, J=6.4 \mathrm{~Hz}, 1 \mathrm{H}), 1.18(\mathrm{dd}, J=9.7,6.3 \mathrm{~Hz}, 1$ H), 1.72 (br q, $J=8.0 \mathrm{~Hz}, 1 \mathrm{H}), 1.90-2.04(\mathrm{~m}, 1 \mathrm{H}), 2.12-2.25(\mathrm{~m}, 1 \mathrm{H}), 2.35-2.60(\mathrm{~m}, 2 \mathrm{H})$, 5.02-5.14 (m, 2 H), 5.47 (ddd, J = 17.0, 10.3, 8.2 Hz, 1 H), 7.35 (br s, $1 \mathrm{H}$ ).

${ }^{13} \mathrm{C}$ NMR (63 MHz, $\left.\mathrm{CDCl}_{3}\right): \delta(\mathrm{ppm}) 17.6\left(\mathrm{CH}_{2}\right), 24.6\left(\mathrm{CH}_{2}\right), 25.4(\mathrm{CH}), 31.0\left(\mathrm{CH}_{2}\right), 44.8$ (C), $115.3\left(\mathrm{CH}_{2}\right), 135.6(\mathrm{CH}), 177.8(\mathrm{C})$.

MS (EI): m/z (\%) $137\left(\mathrm{M}^{+\bullet}, 100\right), 108$ (24), 94 (51), 80 (21), 53 (66).

\section{3 - Mixture of 4-Aza-1-methyl-1-vinylspiro[2.4]heptan-5-one (4b) and 4-Aza-1-(prop-1-}

\section{en-2-yl)-spiro[2.4]heptan-5-one (4c)}

Reaction performed in $\mathrm{Et}_{2} \mathrm{O}: 40 \%$ yield of $\mathbf{4 b}$ (90:10 mixture of diastereomers) and $21 \%$ yield of $\mathbf{4 c}$ (74:26 mixture of diastereomers).

Reaction performed in THF: $44 \%$ yield of $\mathbf{4 b}$ ( $>98: 2$ mixture of diastereomers) and $24 \%$ yield of $\mathbf{4 c}(97: 3$ mixture of diastereomers).

IR (film): $v\left(\mathrm{~cm}^{-1}\right)$ 3188, 3089, 1696, 1453, 1370.

HRMS (ES): m/z (\%) $152.1077\left(\mathrm{MH}^{+} \mathrm{C}_{9} \mathrm{H}_{14} \mathrm{NO}\right.$ requires 152.1075).

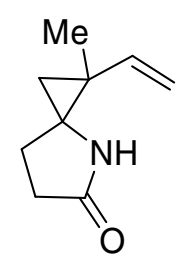

4b (major isomer): ${ }^{1} \mathrm{H}$ NMR $\left(500 \mathrm{MHz}, \mathrm{CDCl}_{3}\right): \delta(\mathrm{ppm}) 0.71(\mathrm{~d}, J=6.1 \mathrm{~Hz}, 1 \mathrm{H}), 1.06(\mathrm{~d}, J$ $=6.1 \mathrm{~Hz}, 1 \mathrm{H}), 1.18(\mathrm{~s}, 3 \mathrm{H}), 1.97(\mathrm{ddd}, J=12.9,9.9,5.4 \mathrm{~Hz}, 1 \mathrm{H}), 2.32(\mathrm{ddd}, J=12.9,9.8$, $7.4 \mathrm{~Hz}, 1 \mathrm{H}), 2.41-2.53(\mathrm{~m}, 2 \mathrm{H}), 5.08$ (dd, $J=17.1,1.2 \mathrm{~Hz}, 1 \mathrm{H}), 5.11(\mathrm{dd}, J=10.7,1.2 \mathrm{~Hz}, 1$ H), $5.74(\mathrm{dd}, J=17.1,10.7 \mathrm{~Hz}, 1 \mathrm{H}), 7.66$ (br s, $1 \mathrm{H})$. 
${ }^{13} \mathrm{C}$ NMR $\left(125 \mathrm{MHz}, \mathrm{CDCl}_{3}\right): \delta(\mathrm{ppm}) 17.6\left(\mathrm{CH}_{3}\right), 24.8\left(\mathrm{CH}_{2}\right), 25.3(\mathrm{C}), 25.6\left(\mathrm{CH}_{2}\right), 30.9$ $\left(\mathrm{CH}_{2}\right), 48.4(\mathrm{C}), 113.3\left(\mathrm{CH}_{2}\right), 140.3(\mathrm{CH}), 177,9(\mathrm{C})$.

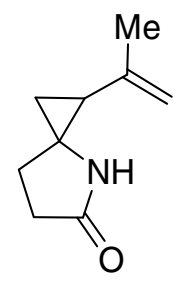

$\underline{4 \mathbf{c} \text { (major isomer): }}{ }^{1} \mathrm{H} \mathrm{NMR}\left(500 \mathrm{MHz}, \mathrm{CDCl}_{3}\right): \delta(\mathrm{ppm}) 0.92(\mathrm{dd}, J=9.1,6.1 \mathrm{~Hz}, 1 \mathrm{H}), 1.07$ $(\mathrm{t}, J=6.8 \mathrm{~Hz}, 1 \mathrm{H}), 1.80(\mathrm{~s}, 3 \mathrm{H}), 2.12$ (ddd, $J=12.8,9.3,5.6 \mathrm{~Hz}, 1 \mathrm{H}), 2.25$ (ddd, $J=12.8$, 9.1, 8.3 Hz, 1 H), 2.44-2.53 (m, 2 H), 4.67 (s, 1 H), 4.89 (s, 1 H), 6.45 (br s, 1 H).

${ }^{13} \mathrm{C}$ NMR (125 MHz, $\left.\mathrm{CDCl}_{3}\right): \delta(\mathrm{ppm}) 14.1\left(\mathrm{CH}_{2}\right), 24.0\left(\mathrm{CH}_{3}\right), 29.2(\mathrm{CH}), 30.1\left(\mathrm{CH}_{2}\right), 31.1$ $\left(\mathrm{CH}_{2}\right), 44.1(\mathrm{C}), 110.3\left(\mathrm{CH}_{2}\right), 141.3(\mathrm{CH}), 177,7(\mathrm{C})$.

\section{4 - Spiro[1-vinylcyclopropane-2,3'-(2',3'-dihydro-1'-isoindolone)] (4d)}

Reaction performed in $\mathrm{Et}_{2} \mathrm{O}: 85 \%$ yield (70:30 mixture of diastereomers)

Reaction performed in THF: $81 \%$ yield (70:30 mixture of diastereomers)

The two diastereoisomers were separated by chromatography (silica gel $-\mathrm{Et}_{2} \mathrm{O}$ )

\section{$\underline{\left(1 R^{*}, 2 R^{*}\right)-4 \mathrm{~d}}$}

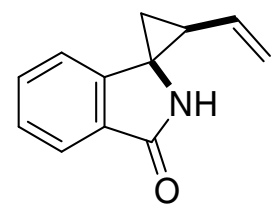

White solid. Mp $171-173{ }^{\circ} \mathrm{C}$

$\mathrm{R}_{f} 0.48$ (Silica gel, $\mathrm{Et}_{2} \mathrm{O}$ )

IR (KBr): $v\left(\mathrm{~cm}^{-1}\right) 3244,1688,1650,1475,1326$.

${ }^{1} \mathrm{H}$ NMR (500 MHz, $\mathrm{CDCl}_{3}$ ): $\delta$ (ppm) 1.75-1.80 (m, $2 \mathrm{H}$ ), 2.37 (q, $\left.J=8.3 \mathrm{~Hz}, 1 \mathrm{H}\right), 5.19$ (dd, $\mathrm{J}=10.3,0.7 \mathrm{~Hz}, 1 \mathrm{H}), 5.25(\mathrm{~d}, J=17.0 \mathrm{~Hz}, 1 \mathrm{H}), 5.88(\mathrm{ddd}, J=17.0,10.3,8.1 \mathrm{~Hz}, 1 \mathrm{H}), 7.10$ $(\mathrm{d}, J=7.7 \mathrm{~Hz}, 1 \mathrm{H}), 7.44(\mathrm{td}, J=7.5,0.8 \mathrm{~Hz}, 1 \mathrm{H}), 7.56(\mathrm{td}, J=7.5,1.0 \mathrm{~Hz}, 1 \mathrm{H}), 7.88(\mathrm{~d}, J=$ $7.5 \mathrm{~Hz}, 1 \mathrm{H}), 8.45$ (br s, $1 \mathrm{H})$.

${ }^{13} \mathrm{C}$ NMR (125 MHz, $\left.\mathrm{CDCl}_{3}\right): \delta(\mathrm{ppm}) 18.8\left(\mathrm{CH}_{2}\right), 29.9(\mathrm{CH}), 46.9(\mathrm{C}), 117.1\left(\mathrm{CH}_{2}\right), 118.0$ $(\mathrm{CH}), 123.9(\mathrm{CH}), 127.4(\mathrm{CH}), 131.6(\mathrm{C}), 131.9(\mathrm{CH}), 134.6(\mathrm{CH}), 148.1(\mathrm{C}), 171.2(\mathrm{C})$. MS (EI): m/z (\%) $185\left(\mathrm{M}^{+\bullet}, 93\right), 156$ (100), 103 (42).

HRMS (ES): m/z (\%) $208.0736\left(\mathrm{MNa}^{+} \mathrm{C}_{12} \mathrm{H}_{11} \mathrm{NONa}\right.$ requires 208.0738). 


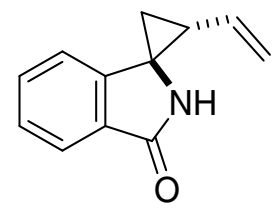

White solid. Mp $122-123^{\circ} \mathrm{C}$

$\mathrm{R}_{f} 0.22$ (Silica gel, $\mathrm{Et}_{2} \mathrm{O}$ )

IR (KBr): $v\left(\mathrm{~cm}^{-1}\right)$ 3189, 1704, 1649, 1473, 1329.

${ }^{1} \mathrm{H}$ NMR (500 MHz, $\mathrm{CDCl}_{3}$ ): $\delta(\mathrm{ppm}) 1.64(\mathrm{dd}, J=7.9,6.5 \mathrm{~Hz}, 1 \mathrm{H}), 2.00(\mathrm{dd}, J=9.7,6.5$

$\mathrm{Hz}, 1 \mathrm{H}), 2.58(\mathrm{q}, J=8.1 \mathrm{~Hz}, 1 \mathrm{H}), 5.22(\mathrm{~d}, J=10.3 \mathrm{~Hz}, 1 \mathrm{H}), 5.29(\mathrm{~d}, J=17.0 \mathrm{~Hz}, 1 \mathrm{H}), 5.86$ (ddd, $J=17.0,10.3,6.9 \mathrm{~Hz}, 1 \mathrm{H}), 7.17$ (d, $J=7.6 \mathrm{~Hz}, 1 \mathrm{H}), 7.44$ (t, $J=7.4 \mathrm{~Hz}, 1 \mathrm{H}), 7.52$ (td, $J=7.5,1.1 \mathrm{~Hz}, 1 \mathrm{H}), 7.89(\mathrm{~d}, J=7.6 \mathrm{~Hz}, 1 \mathrm{H}), 8.75$ (br s, $1 \mathrm{H})$.

${ }^{13} \mathrm{C}$ NMR (125 MHz, $\left.\left.\mathrm{CDCl}_{3}\right)\right): \delta(\mathrm{ppm}) 19.1\left(\mathrm{CH}_{2}\right), 29.2(\mathrm{CH}), 46.6(\mathrm{C}), 118.0\left(\mathrm{CH}_{2}\right), 120.7$ (CH), $123.8(\mathrm{CH}), 127.3(\mathrm{CH}), 131.1(\mathrm{C}), 132.7(\mathrm{CH}), 133.4(\mathrm{CH}), 145.3(\mathrm{C}), 170.8(\mathrm{C})$. MS (EI): m/z (\%) $185\left(\mathrm{M}^{+\bullet}, 100\right), 156$ (80), 103 (14).

HRMS (ES): $\mathrm{m} / \mathrm{z}(\%) 208.0741\left(\mathrm{MNa}^{+} \mathrm{C}_{12} \mathrm{H}_{11} \mathrm{NONa}\right.$ requires 208.0738).

\section{5 - 4-Aza-1-vinylspiro[2.5] octan-5-one (4e)}

Reaction performed in $\mathrm{Et}_{2} \mathrm{O}: 75 \%$ yield (70:30 mixture of diastereomers)

Reaction performed in THF: 77\% yield (63:37 mixture of diastereomers)

The two diastereomers were separated by chromatography (silica gel $-\mathrm{CH}_{2} \mathrm{Cl}_{2} / i$ - $\mathrm{PrOH}$ 95:5)

\section{$\underline{\left(1 R^{*}, 3 R^{*}\right)-4 \mathrm{e}}$}

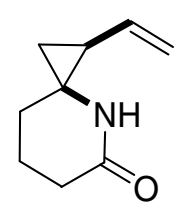

White solid. Mp $138-139^{\circ} \mathrm{C}$

$\mathrm{R}_{f} 0.47$ (Silica gel, $\mathrm{CH}_{2} \mathrm{Cl}_{2} / i-\mathrm{PrOH}$ 95:5)

IR (KBr): v $\left(\mathrm{cm}^{-1}\right)$ 3176, 3061, 1656, 1400.

${ }^{1} \mathrm{H}$ NMR $\left(250 \mathrm{MHz}, \mathrm{CDCl}_{3}\right): \delta(\mathrm{ppm}) 0.84(\mathrm{t}, J=6.3 \mathrm{~Hz}, 1 \mathrm{H}), 1.03(\mathrm{dd}, J=9.0,6.1 \mathrm{~Hz}, 1$ H), 1.52-1.68 (m, 2 H), 1.75-1.98 (m, 3 H), $2.42(\mathrm{t}, J=6.7 \mathrm{~Hz}, 1 \mathrm{H}), 5.08-5.20$ (m, $2 \mathrm{H}), 5.70$ (ddd, $J=17.4,9.9,7.5 \mathrm{~Hz}, 1 \mathrm{H}), 6.31$ (br s, $1 \mathrm{H}$ ). 
${ }^{13} \mathrm{C} \mathrm{NMR}\left(63 \mathrm{MHz}, \mathrm{CDCl}_{3}\right): \delta(\mathrm{ppm}) 18.3\left(\mathrm{CH}_{2}\right), 19.8\left(\mathrm{CH}_{2}\right), 27.6(\mathrm{CH}), 31.2\left(\mathrm{CH}_{2}\right), 32.2$ $\left(\mathrm{CH}_{2}\right), 40.9(\mathrm{C}), 116.5\left(\mathrm{CH}_{2}\right), 134.6(\mathrm{CH}), 173.2(\mathrm{C})$.

MS (EI): m/z (\%) $151\left(\mathrm{M}^{+\bullet}, 100\right), 149$ (64), 108 (77).

HRMS (ES): m/z (\%) $152.1072\left(\mathrm{MH}^{+} \mathrm{C}_{9} \mathrm{H}_{14} \mathrm{NO}\right.$ requires 152.1072).

\section{$\underline{\left(1 R^{*}, 3 S^{*}\right)-4 \mathrm{e}}$}

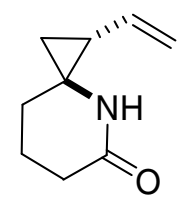

White solid. Mp $105-106^{\circ} \mathrm{C}$

$\mathrm{R}_{f} 0.37$ (Silica gel, $\mathrm{CH}_{2} \mathrm{Cl}_{2} / i-\mathrm{PrOH}$ 95:5)

IR (KBr): v $\left(\mathrm{cm}^{-1}\right)$ 3175, 3079, 1664, 1629, 1393.

${ }^{1} \mathrm{H}$ NMR $\left(250 \mathrm{MHz}, \mathrm{CDCl}_{3}\right): \delta(\mathrm{ppm}) 0.77(\mathrm{t}, J=6.4 \mathrm{~Hz}, 1 \mathrm{H}), 1.07(\mathrm{dd}, J=9.4,6.1 \mathrm{~Hz}, 1$ H), 1.60-1.87 (m, $5 \mathrm{H}), 2.37(\mathrm{t}, J=6.5 \mathrm{~Hz}, 1 \mathrm{H}), 5.02-5.15(\mathrm{~m}, 2 \mathrm{H}), 5.55(\mathrm{ddd}, J=17.0,9.9$, $8.0 \mathrm{~Hz}, 1 \mathrm{H}), 7.30$ (br s, $1 \mathrm{H})$.

${ }^{13} \mathrm{C} \mathrm{NMR}\left(63 \mathrm{MHz}, \mathrm{CDCl}_{3}\right): \delta(\mathrm{ppm}) 18.8\left(\mathrm{CH}_{2}\right), 19.6\left(\mathrm{CH}_{2}\right), 26.4(\mathrm{CH}), 28.1\left(\mathrm{CH}_{2}\right), 31.1$

$\left(\mathrm{CH}_{2}\right), 41.1(\mathrm{C}), 116.0\left(\mathrm{CH}_{2}\right), 135.0(\mathrm{CH}), 173.3(\mathrm{C})$.

MS (EI): m/z (\%) $151\left(\mathrm{M}^{+\bullet}, 63\right), 149$ (71), 108 (52), 94 (100).

HRMS (ES): m/z (\%) $152.1074\left(\mathrm{MH}^{+} \mathrm{C}_{9} \mathrm{H}_{14} \mathrm{NO}\right.$ requires 152.1072).

\section{$\underline{3.6-\left(1 R^{*}, 3 R^{*}\right) \text { - 4-Aza-6-oxa-1-vinylspiro[2.4]heptan-5-one (4f) }}$}

Reaction performed in THF: $57 \%$ yield ( $>98: 2$ mixture of diastereomers)

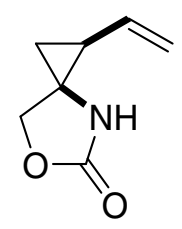

Pale yellow oil.

IR (film): $v\left(\mathrm{~cm}^{-1}\right)$ 3262, 1749, 1639, 1392, 1270.

${ }^{1} \mathrm{H}$ NMR (250 MHz, $\mathrm{CDCl}_{3}$ ): $\delta$ (ppm) 0.94-1.04 (m, $\left.2 \mathrm{H}\right), 1.54$ (q, J=8.2 Hz, $\left.1 \mathrm{H}\right), 4.31$ (s, 2 H), $5.07(\mathrm{~d}, J=10.1 \mathrm{~Hz}, 1 \mathrm{H}), 5.12(\mathrm{~d}, J=17.0 \mathrm{~Hz}, 1 \mathrm{H}), 5.50(\mathrm{ddd}, J=17.0,10.1,8.2 \mathrm{~Hz}, 1$ H), 7.30 (br s, $1 \mathrm{H})$.

${ }^{13} \mathrm{C} \mathrm{NMR}\left(63 \mathrm{MHz}, \mathrm{CDCl}_{3}\right): \delta(\mathrm{ppm}) 15.8\left(\mathrm{CH}_{2}\right), 25.9(\mathrm{CH}), 42.8(\mathrm{C}), 71.4\left(\mathrm{CH}_{2}\right), 116.5$ $\left(\mathrm{CH}_{2}\right), 133.9(\mathrm{CH}), 160.3(\mathrm{C})$. 


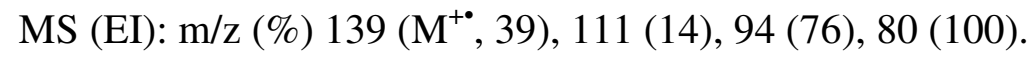

HRMS (ES): $\mathrm{m} / \mathrm{z}(\%) 162.0534\left(\mathrm{MH}^{+} \mathrm{C}_{7} \mathrm{H}_{9} \mathrm{NO}_{2} \mathrm{Na}\right.$ requires 162.0531). 

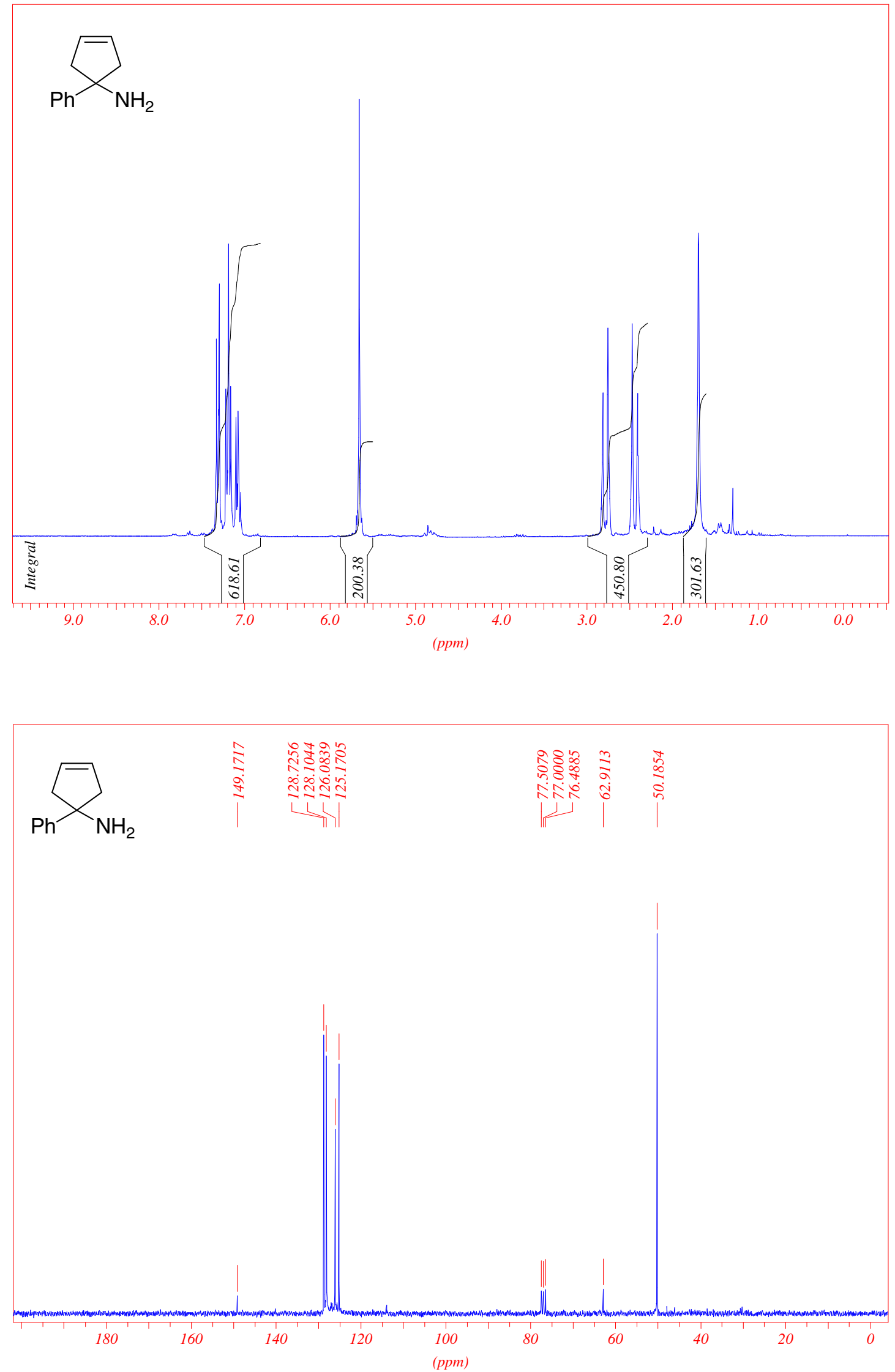

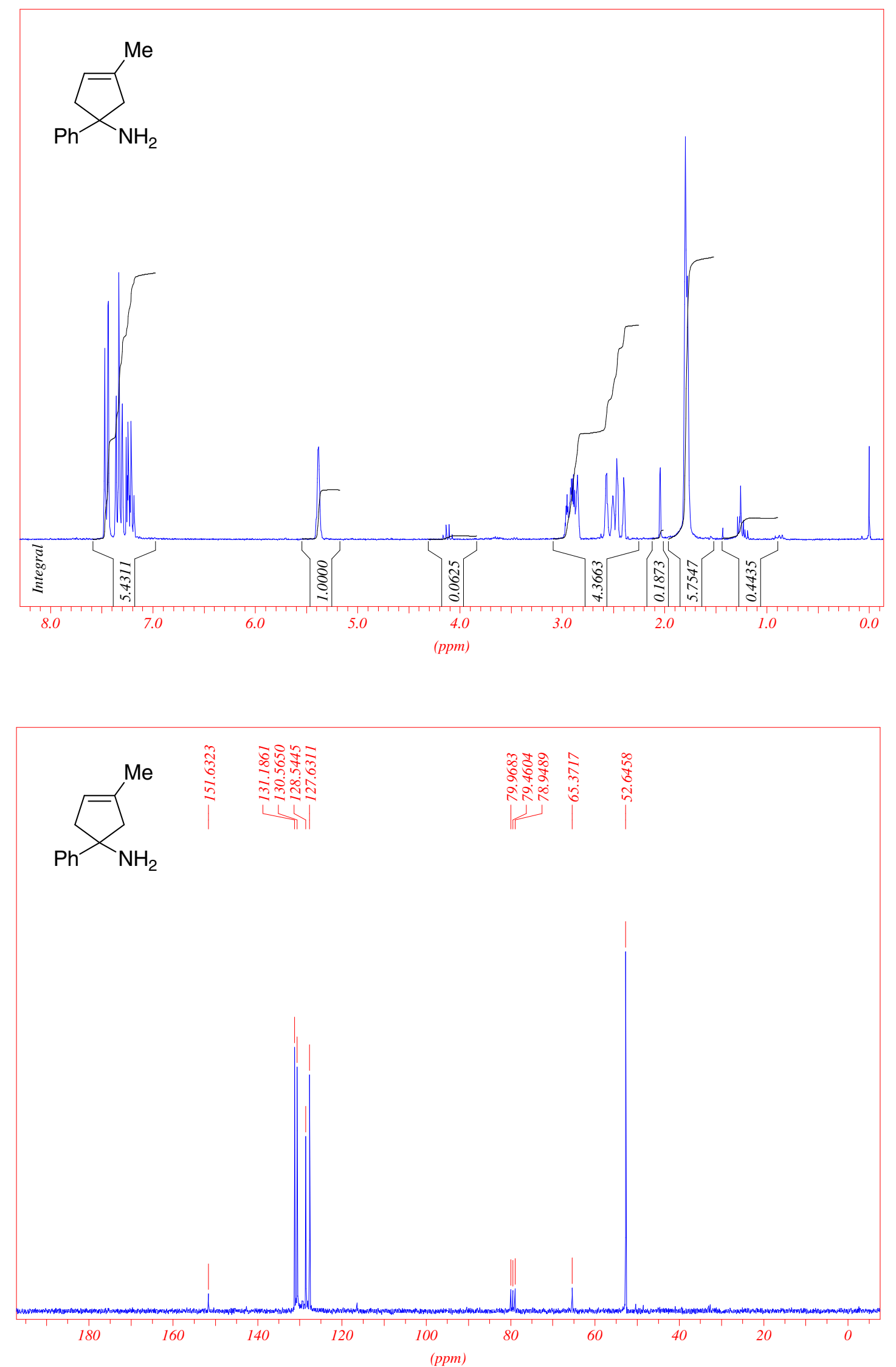


\section{$\underline{\text { (1-nonylcyclopent-3-enyl)amine (1c) }}$}
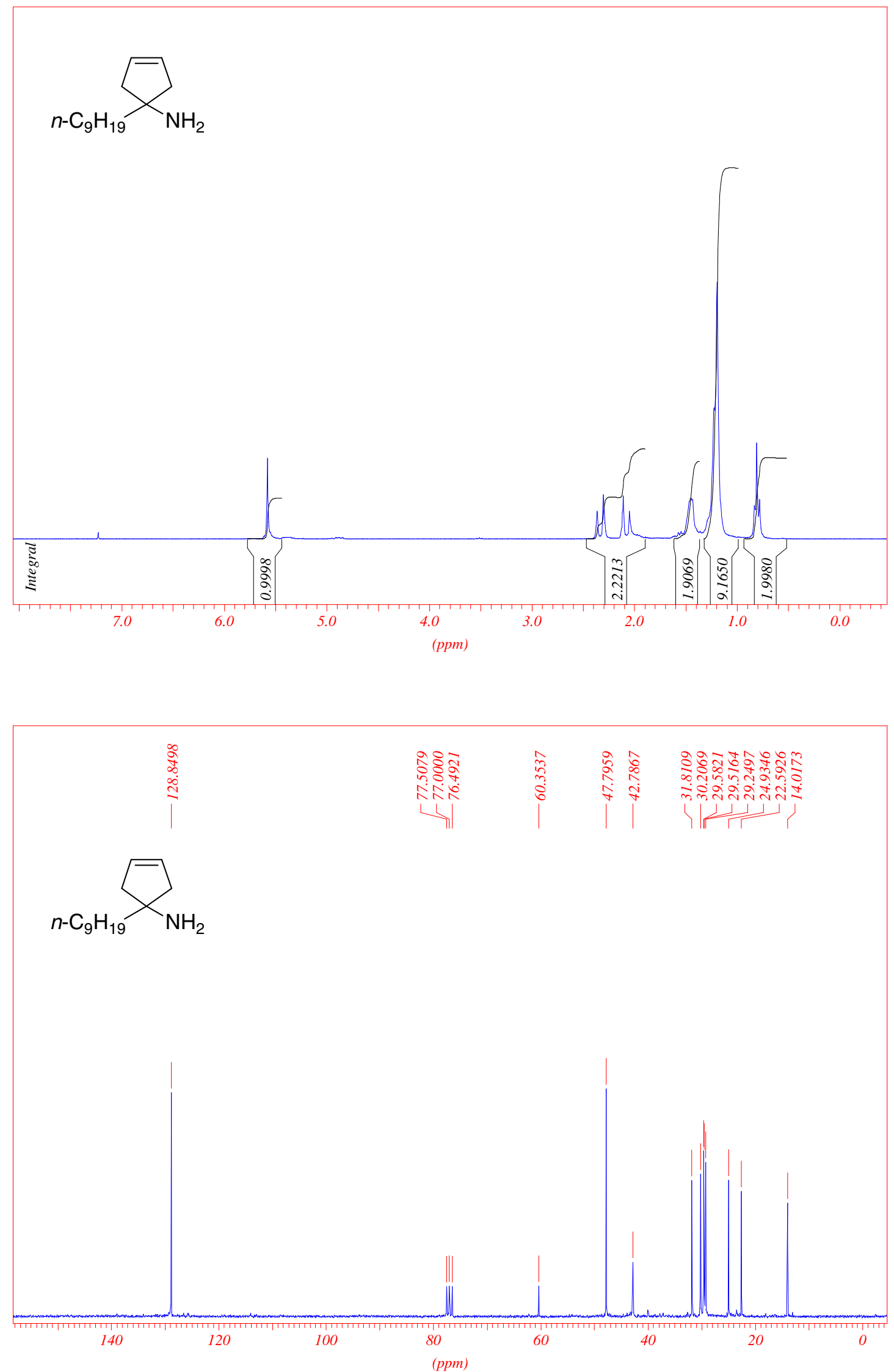
(3-Methyl-1-nonylcyclopent-3-enyl)amine (1d)
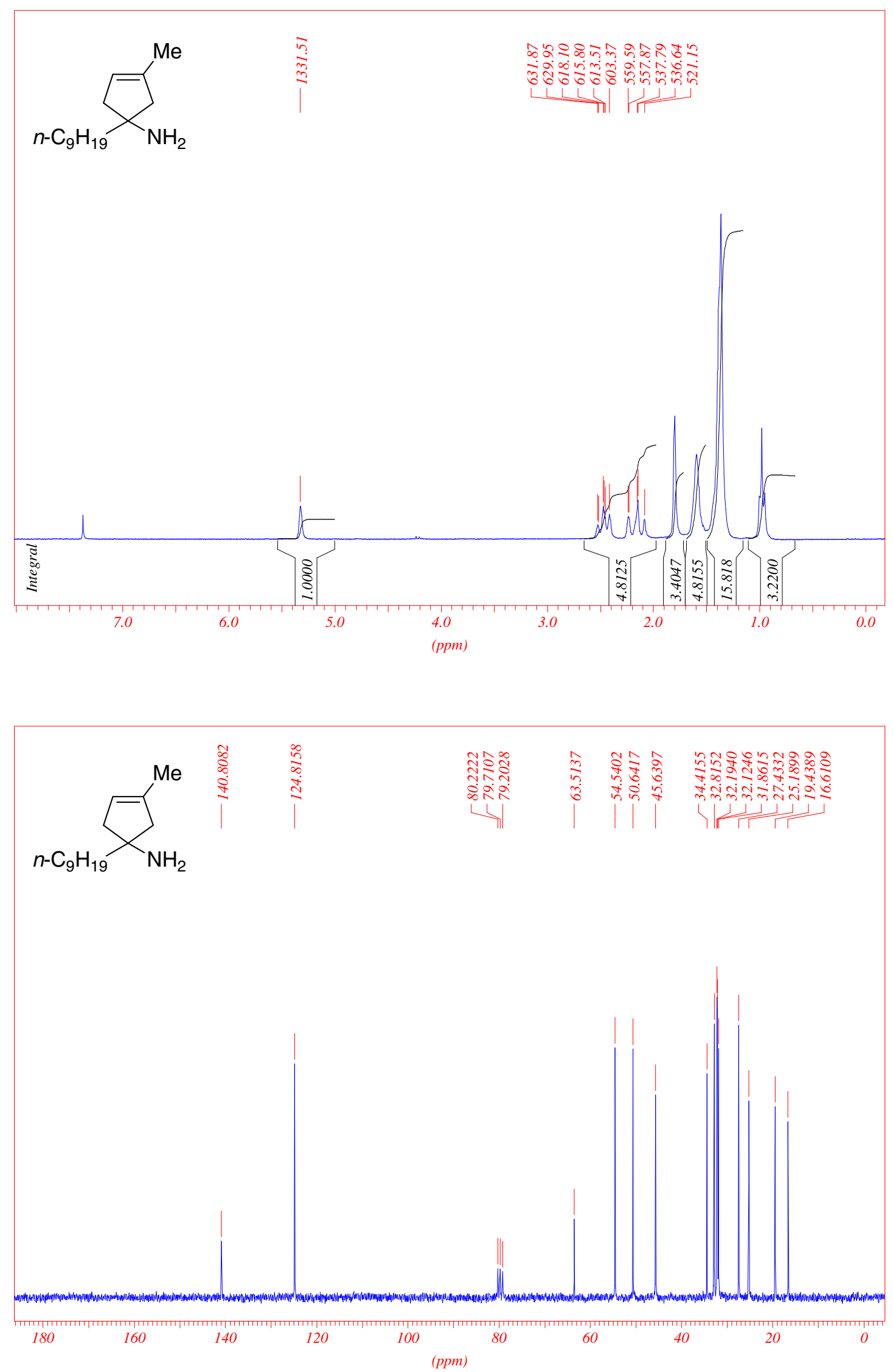

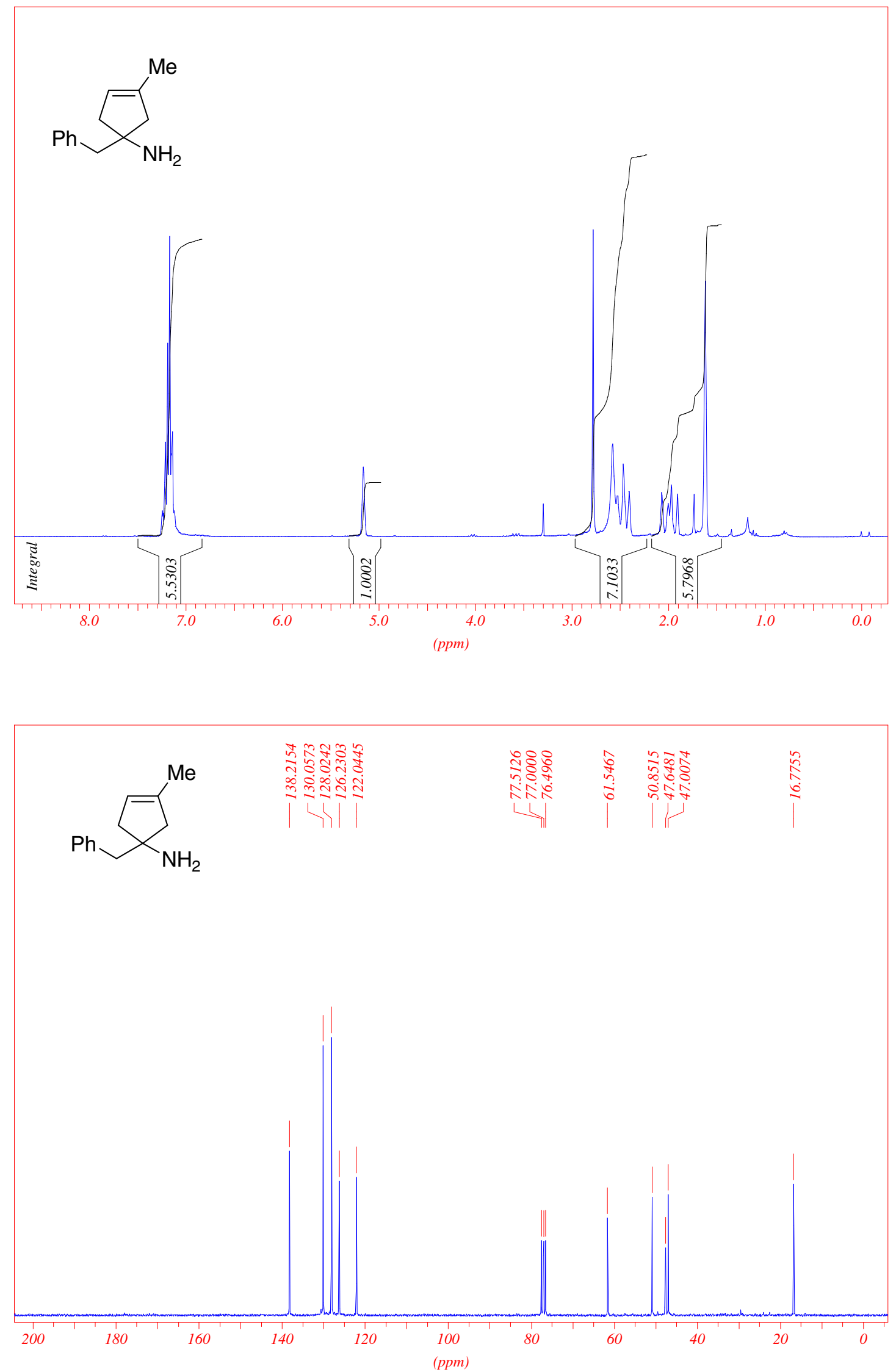

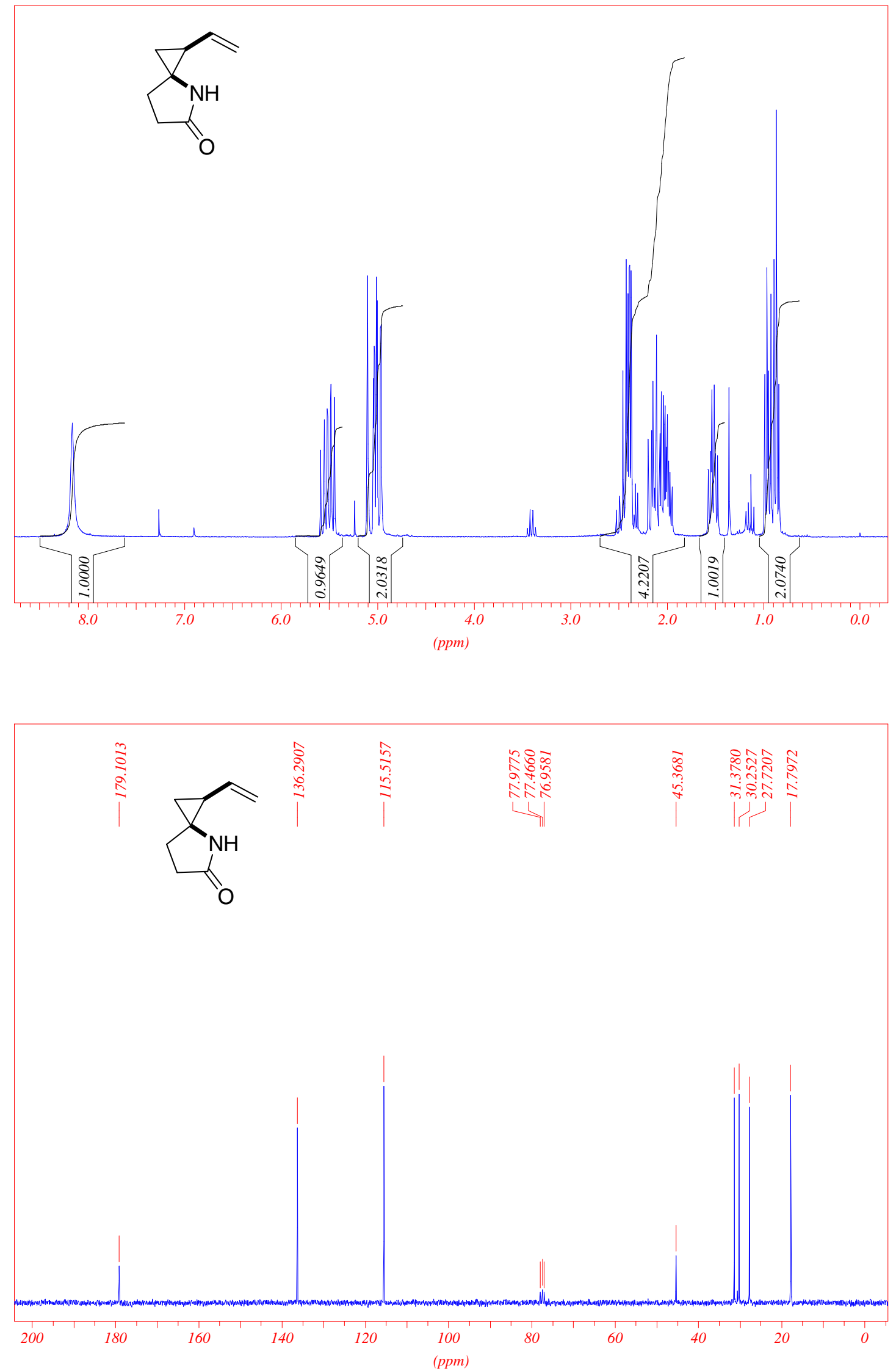

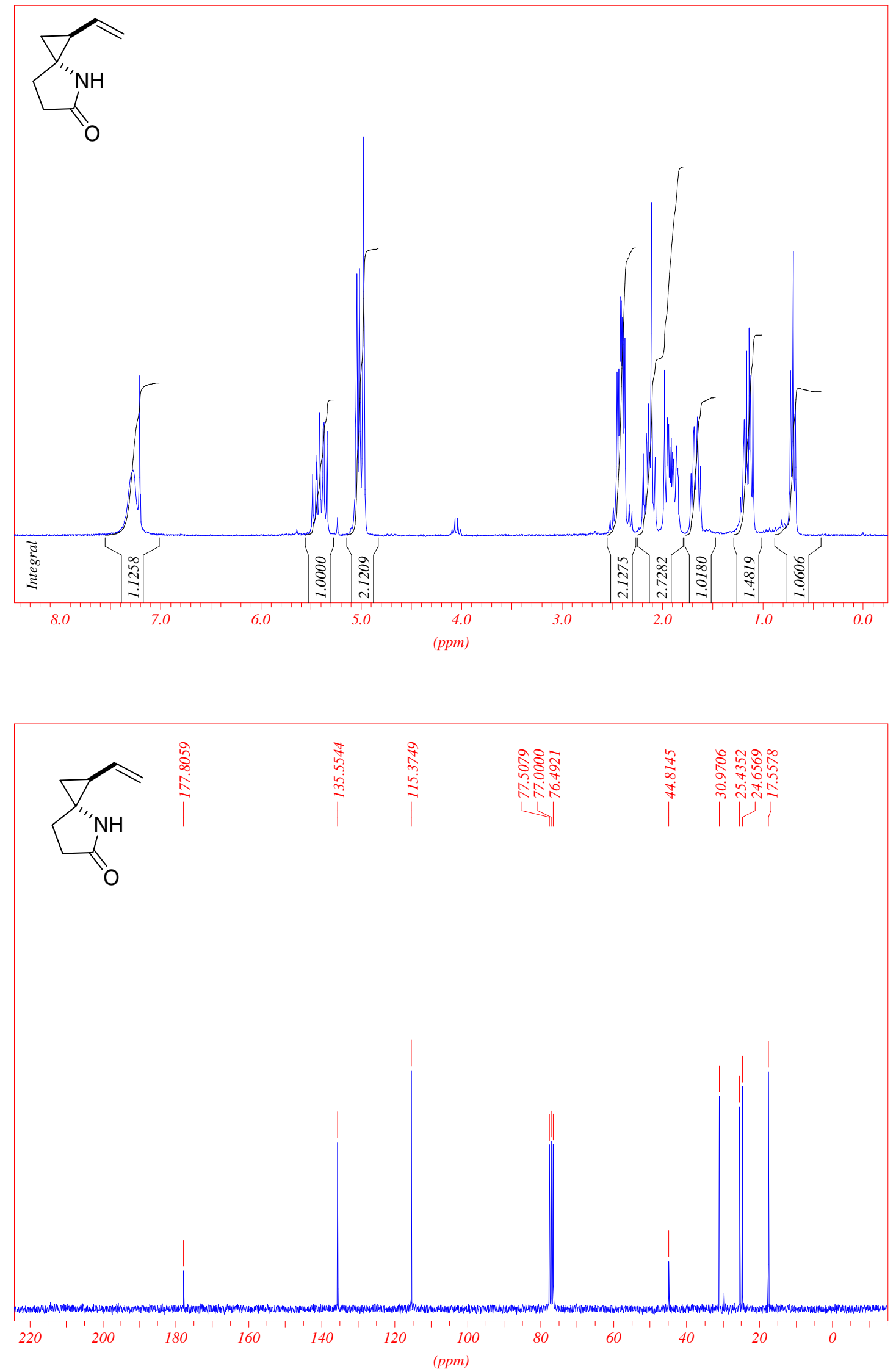

S-16 


\section{spiro[2.4]heptan-5-one (4c)}
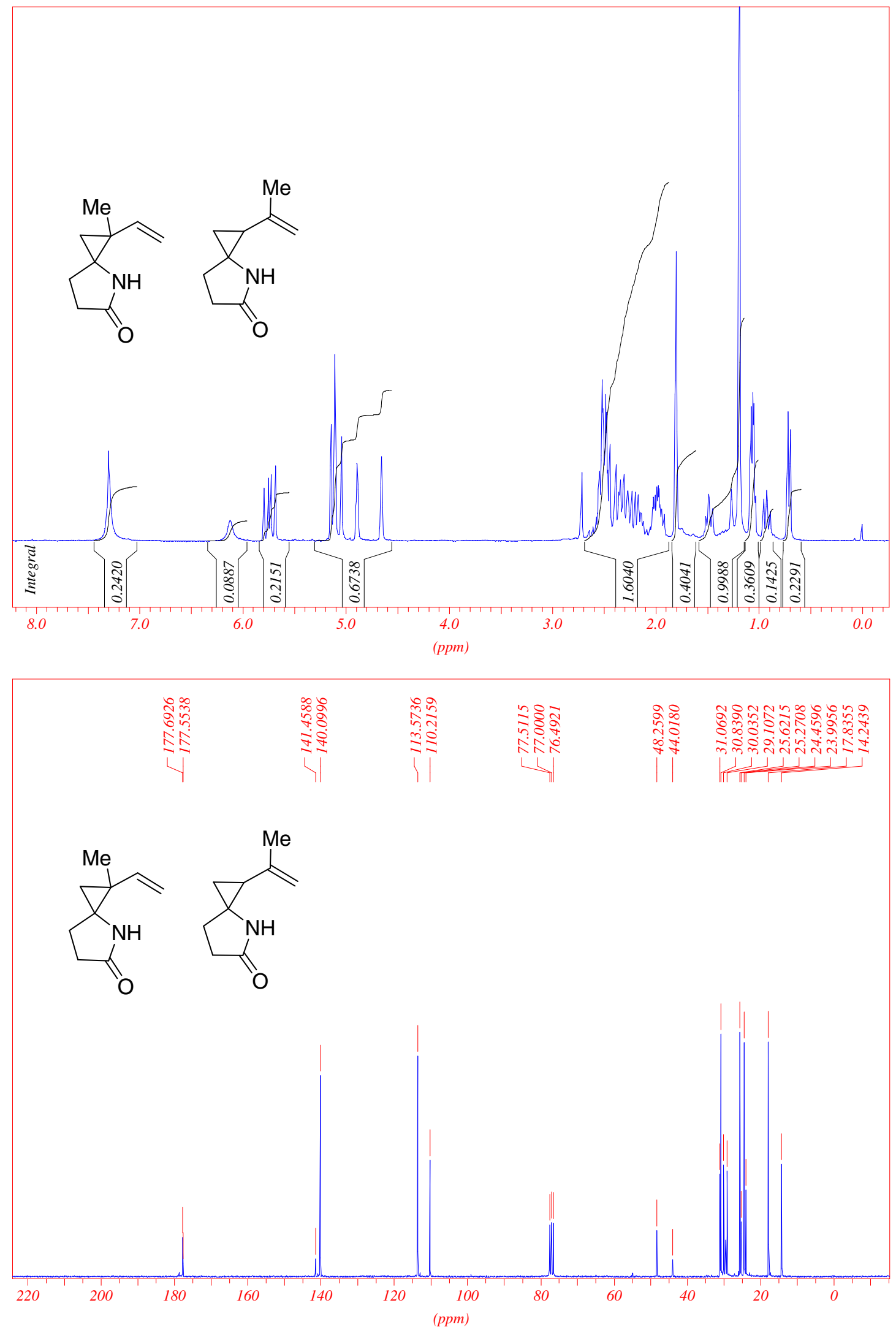

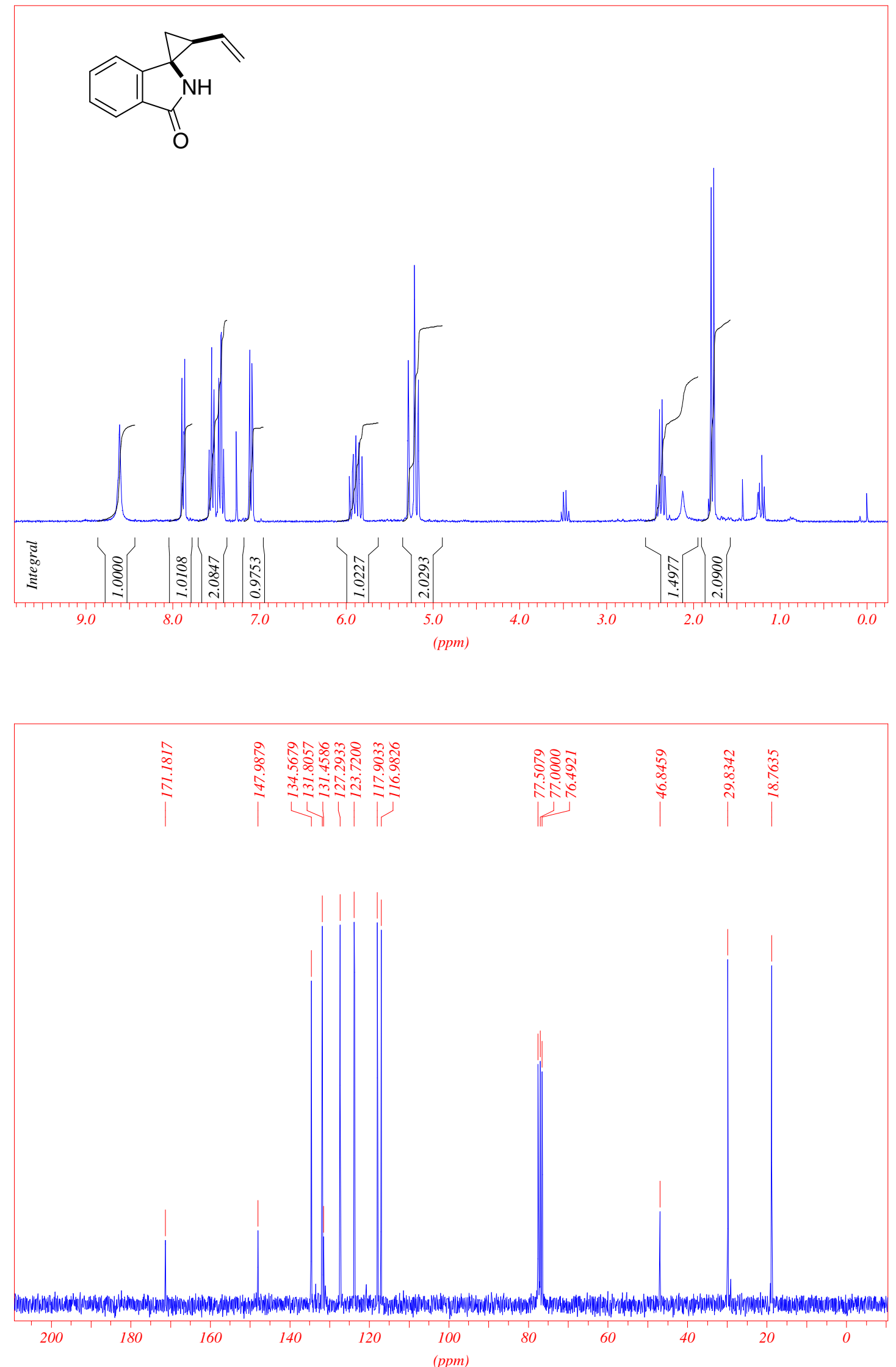

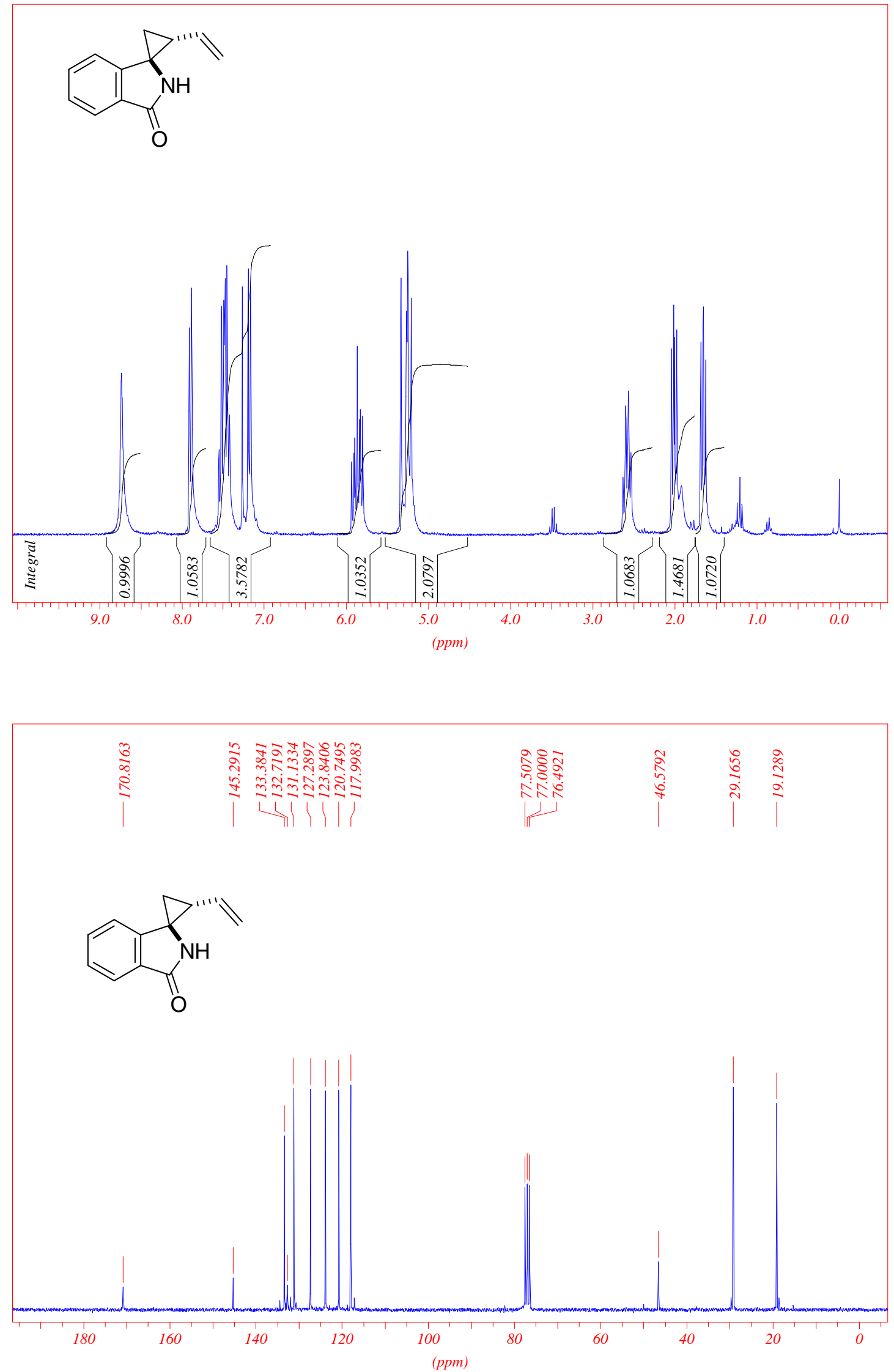

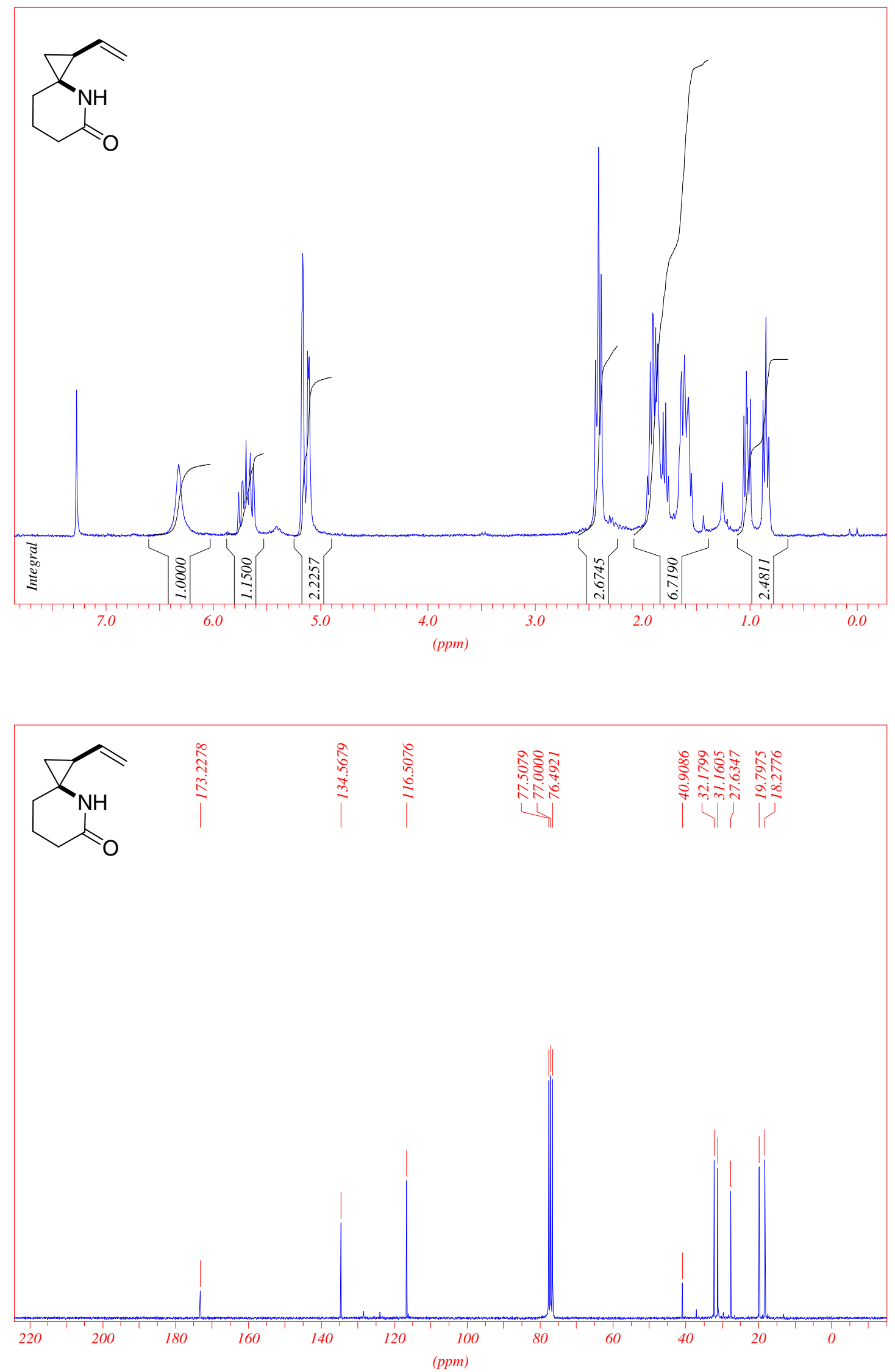

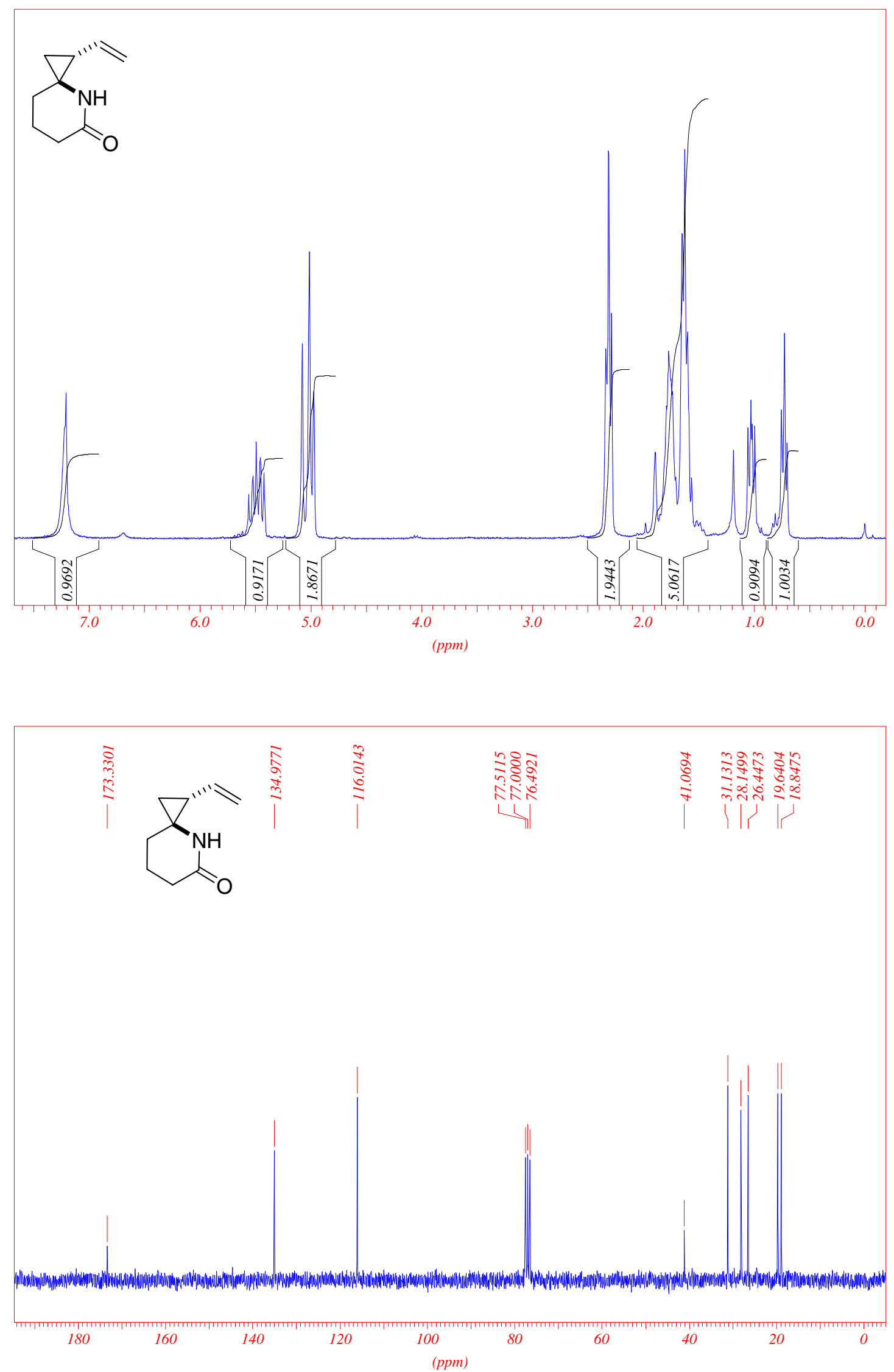

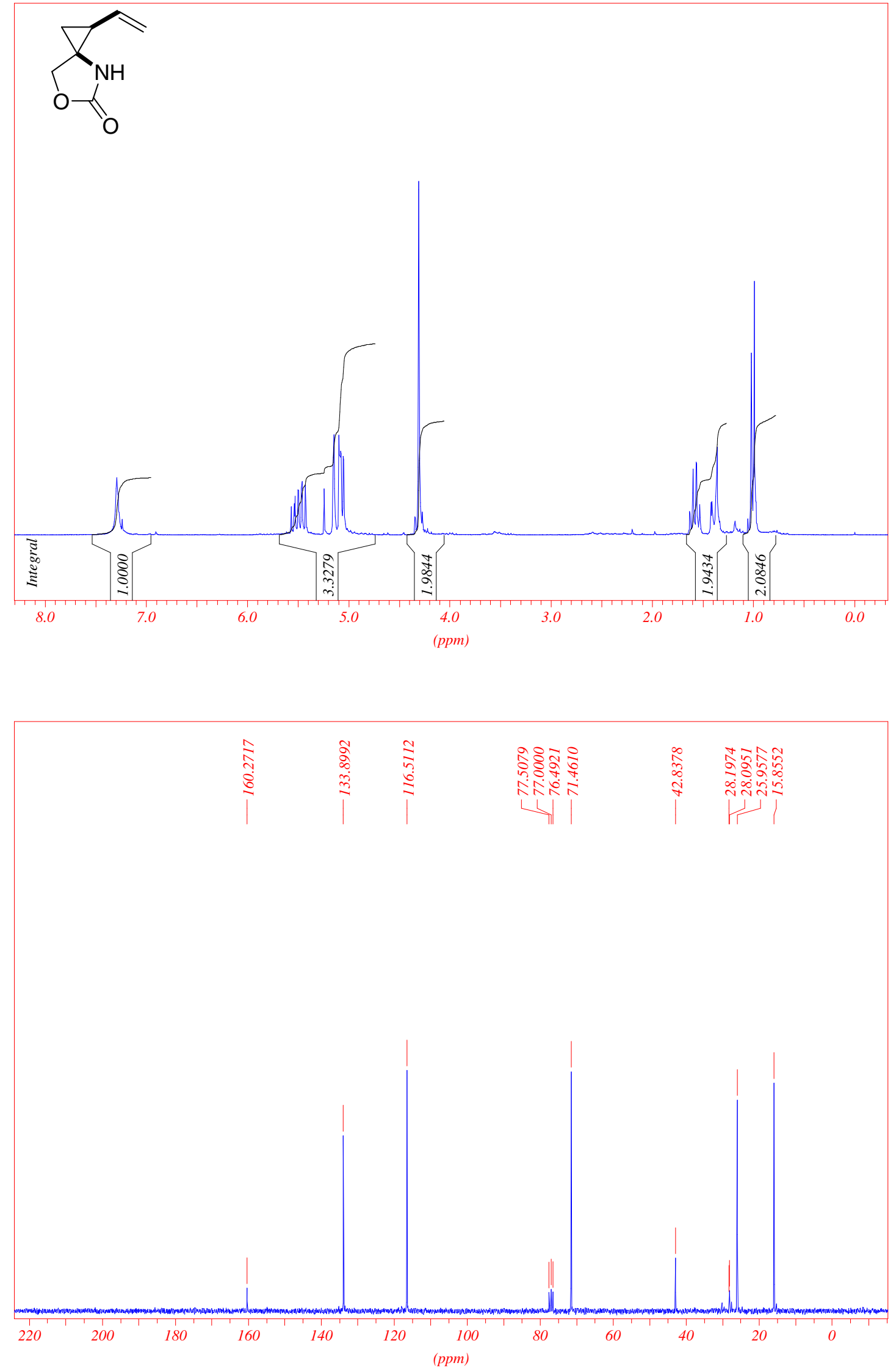